\title{
Intracranial region of the vertebral artery: morphometric study in the context of clinical usefulness
}

\author{
J. Dzierżanowski ${ }^{1}$, A. Szarmach², B. Baścik², P. Czapiewski³ 4, A. Muc ${ }^{5}$, M. Piskunowicz², \\ M. Krakowiak' ${ }^{1}$, T. Szmuda ${ }^{1}$, P. Stoniewski ${ }^{1}$, E. Szurowska ${ }^{2}$, P.J. Winklewski ${ }^{6,7}$
}

${ }^{1}$ Department of Neurosurgery, Medical University of Gdansk, Poland ${ }^{2} 2^{\text {nd }}$ Department of Radiology, Medical University of Gdansk, Poland ${ }^{3}$ Department of Pathomorphology, Medical University of Gdansk, Poland

${ }^{4}$ Department of Pathology, Medical Faculty, Otto-von-Guericke University Magdeburg, Germany

${ }^{5}$ Faculty of Electrical Engineering, Gdynia Maritime University, Poland

6 Institute of Human Physiology, Medical University of Gdansk, Poland

${ }^{7}$ Institute of Health Sciences, Pomeranian University of Slupsk, Poland

[Received: 24 October 2016; Accepted: 29 November 2016]

Background: The aim of this study was to analyse the morphometry of the intracranial segment of the vertebral artery in the context of clinical usefulness. The results were compared with published data available in full-text archived medical journals.

Materials and methods: More than 100 digital subtraction angiography (DSA) and 3-dimensional (3D) angio-computed tomography (CT) examinations were used to measure the following parameters: the whole and partial length of $V_{4}$ in characteristic anatomical points, the diameter in three places (on the level of foramen magnum, in point of exit to the posterior inferior cerebellar artery, and in the vertebro-basilar junction), the angle of connection to the vertebral arteries, and all anatomical variations including fenestration, duplication, dolichoectasia or absent artery.

Results: The left $V_{4}$ section was predominant over the right artery, which is manifested by length, width, cases of ectasia and fewer cases of hypoplasia. The incidences of $V_{4}$ ectasia were identified more often than those documented in the accessible literature, and they were found in the natural location of formation of saccular aneurysms.

Conclusions: The presented knowledge of anatomical variation and abnormalities of vertebral circulation can improve the accuracy and "safety" of the surgical procedures in this region, help to determine the range of surgical approach and avoid associated complications. The radiological examinations using 3D CT, DSA reveal unlimited observation of anatomical structures in contrast to studies based on cadavers, and can complement the morphometry in anatomical preparations. (Folia Morphol 2017; 76, 3: 379-387)

Key words: vertebral artery, hypoplasia, morphometry, dolichoectasia, vertebro-basilar junction

Address for correspondence: P. Winklewski, MD, PhD, Institute of Human Physiology, Medical University of Gdansk, ul. Tuwima 15, 80-210 Gdańsk, Poland, tel/fax: +48 5834915 15, e-mail: pawelwinklewski@wp.pl 


\section{INTRODUCTION}

The intracranial segment of the vertebral artery (VA) begins at the foramen magnum and finishes in the joint with the contralateral artery usually at the level of the pontomedullary junction, facing the inferior clivus. It ascends anterior to the dentate ligament and the accessory nerve, and anterior or between the hypoglossal rootless to reach the anterior surfaces of the medulla oblongata. The following branches arise from the $\mathrm{V}_{4}$ : anterior and posterior spinal arteries, anterior and posterior meningeal arteries and the posterior inferior cerebellar artery.

The basis of this arterial anatomy is well known; however, the high frequency of anatomical anomalies necessitates the description of a circumstantial morphometry of the $\mathrm{V}_{4}$ segment with its anatomical variability in the context of clinical usefulness (radiology, neurosurgery, head and facial surgery, otolaryngology, vascular surgery). In this study, we analysed all of the details regarding the anatomical variations of the VA complex on the basis of radiological vascular examinations. The salient matters of these anomalies and normal variations were discussed with relevance to clinical practice. In addition, we compared our results with data available in full-text archived medical journals. In our opinion, this is the most comprehensive analysis of the morphometry of the intracranial regions of the VA. This knowledge is very helpful for clinical practice especially for vascular neurosurgeons and radiologists.

\section{MATERIALS AND METHODS}

In this study, 104 digital subtraction angiography (DSA) examinations and 104 three-dimensional (3D) computed tomography (CT) angiography (CTA) images were used to obtain the information about $V_{4}$. These examinations were performed by examining adult people of the Caucasian race ( 37 men and 68 women), and the vessels were measured bilaterally (208 results). All patients from the examined group were diagnosed in the Medical University of Gdansk in the Radiology Department and Neurosurgery Department because of headache, unclear CTA images or subarachnoid haemorrhage (SAH). The experimental protocol and the study were approved by the ethical committee of the Medical University of Gdansk. The CTA exam was the first step taken in the given research group. DSA, as a gold standard, was performed in those patients where vascular pathology was not observed. Patients with double-negative vascular radiological examinations were included in this study. Patients were excluded from the study if they had ischaemic or haemorrhagic attack, focal neurological deficits, and if there were recognised vascular (arterial malformations, aneurysms) or brain tissue pathologies. All of the CTA images were performed with a 64-MDct LightSpeed Vct Xt (GE Healthcare Technologies, Wisconsin, USA). The protocol for circle of Willis CTA included $0.625-\mathrm{mm}$ slice thickness, with a concentration (recons) to $0.2 \mathrm{~mm}$, and cuts were obtained with a tracking marker placed in the aortic arch. The parameters of the examinations included: the angle of the gantry $-0^{\circ}$, the interval $-0.625 \mathrm{~mm}$, rotation time $-0.4 \mathrm{~s}$, pitch $-0.984 \mathrm{~mm}$ in a caudocranial scan direction. The other acquisition parameters were as follows: tube voltage $120 \mathrm{kVp}$, tube current $500 \mathrm{mAs}$, matrix of $512 \times 512$, DFOV $-250 \mathrm{~mm}$, total exposure time $-4.23 \mathrm{~s}$. An amount of $60 \mathrm{~mL}$ of nonionic contrast media (Iomeron 250, Bracco Imaging Deutschland $\mathrm{GmbH}$ ) was injected at $4 \mathrm{~mL} / \mathrm{s}$ through a large-bore intravenous access in the antecubital vein, followed by a chaser bolus of $30 \mathrm{~mL}$ injected at the same rate. Reconstructions of the raw data were performed using a separate workstation (Advantage 4.4; GE Healthcare). Axial images as well as the reconstructed multiplanar (MPR) and maximum intensity projections (MIP) were used primarily for diagnostic purposes. 3D imaging was also used in helping to diagnose patients.

Cerebral angiography was performed by a standard technique by puncturing the right femoral artery and selective catheterisation of both internal carotid and vertebral arteries with an $\mathrm{H} 15 \mathrm{~F}$ catheter (Cook Medical, Bloomington, IN). Angiographies were performed under biplane fluoroscopic guidance (Artis zee biplane system, Siemens, Germany). The CTA and DSA images were evaluated using a PACS workstation (Syngovia, Siemens, Erlangen, Germany).

The following parameters were measured: 1) the whole length of $\mathrm{V}_{4}$ (from entrance to the skull base to the vertebrobasilar junction [VBJ]); 2) the length from the foramen magnum to the posterior inferior cerebellar artery (FM/PICA); 3) the length from the PICA to the VBJ (PICAVBJ); 4) the diameter in three places (on the level of the foramen magnum = FM/NA, in point of exit posterior inferior cerebellar artery = PICANA, and in the VBJ); 5) the angle of connection to the VAs; and 6) all anatomical variations like fenestration, duplication, dolichoectasia or absent artery. The length of $\mathrm{V}_{4}$ and the angle of VBJ were measured using CTA, and the diameters were based on DSA (Figs. 1, 2). All obtained results were analysed separately (left and right side), considering the correlations between sex, age, and sight 


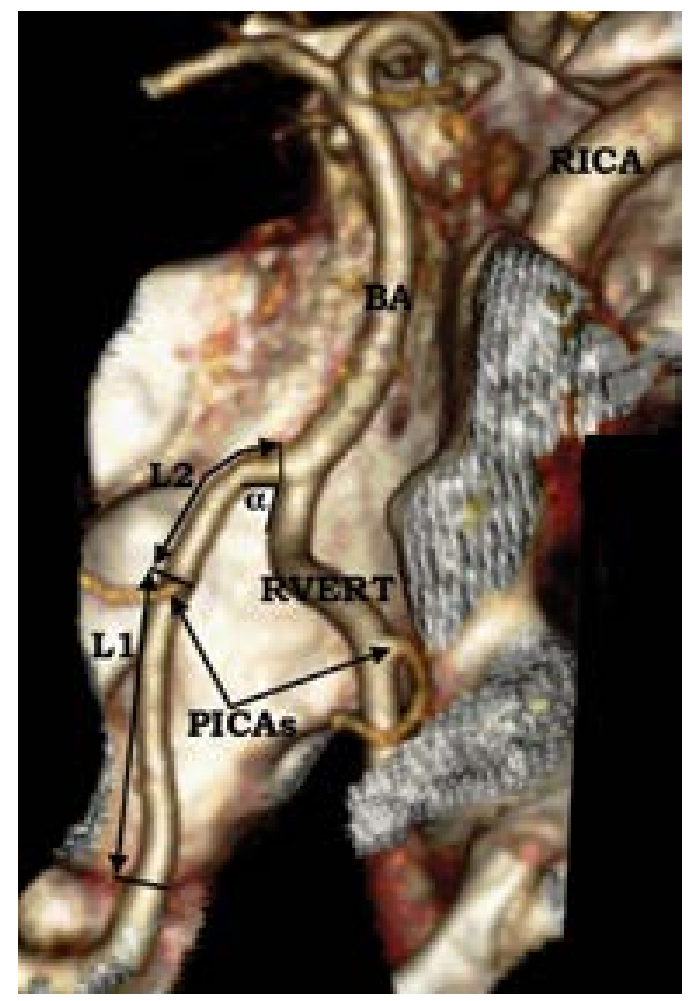

Figure 1. Three-dimensional reconstruction of vertebral artery circulation. Measured variables; $\alpha$ - the angle of connection of the vertebral arteries; $L 1$ - the length of the vertebral artery from the foramen magnum to posterior inferior cerebellar artery (PICA); L2 - the length of the vertebral artery from PICA to the vertebrobasilar junction (VBJ). Whole length of $\mathrm{V}_{4}$ from entrance to the skull base to the VBJ was a sum of $L 1$ and $L 2 ; B A$ - basilar artery; RVERT — right vertebral artery; LVERT — left vertebral artery; RICA — right internal cervical artery.

of measurement, by two independent, experienced (6 and 10 years of experience in performing DSA procedure) radiologists.

\section{Comparative analysis - studies selection}

Medline, Embase, and PubMed database were searched for morphological studies that could be used to compare with obtained results. Only fulltext articles (case reports without systematic review and abstracts were excluded) performed on cadaver preparations and/or radiological examinations were included. All measurements of VAs were performed in adult patients. The papers describing children's anatomy were also excluded.

\section{Statistical analysis}

All measurements were obtained by two independent radiologists and are presented as the mean \pm standard

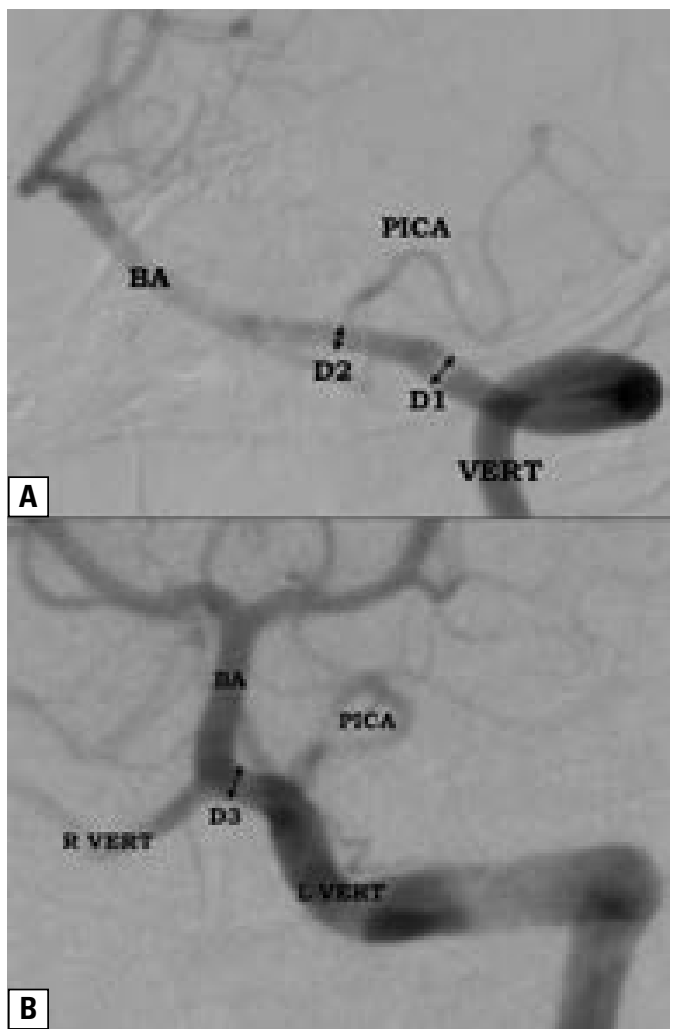

Figure 2. Digital subtraction angiography of the vertebro-basilar circulation. Measured variables; A. D1 - diameter of the vertebral artery at the level of the foramen magnum; D2 - diameter of the vertebral artery at the point of origin of the posterior inferior cerebellar artery (PICA); B. D3 - diameter of the vertebral artery at the level of the vertebro-basilar junction; BA — basilar artery; RVERT — right vertebral artery; LVERT — left vertebral artery.

deviation (SD). For discrete and continuous data, the percentage and median (min-max) values were used. The data obtained were digitized in Excel tables, and the statistical analyses were performed using the STAT 8.0 programme. All parameters of the vessels were described with their means, SD, and the range of the normal variables with their ratios. Statistical analyses included $\chi^{2}$ tests and Student's t-tests, accepting $p>0.005$.

\section{RESULTS}

Demographic results: 66 (63.46\%) females and 38 (36.54\%) males, for a total 104 cases, were included in our study. The mean value of age in the first group was 55.29 ( $22-87$ years old), and 64.95 ( $43-87$ years old) in the second group. The results of measured parameters (length, diameter, angle of VBJ) are presented in Table 1.

The angle of connection to the VAs at the point of the starting basilar artery was from $57^{\circ}$ to $98^{\circ}$; the 
Table 1. The results of morphometry

\begin{tabular}{|c|c|c|c|c|c|c|c|c|c|}
\hline \multirow[t]{2}{*}{ Item } & \multicolumn{4}{|c|}{ Women $(n=66)$} & \multicolumn{4}{|c|}{ Men (n = 38) } & \multirow{2}{*}{$\begin{array}{c}\text { Statistic } \\
\text { test t }\end{array}$} \\
\hline & Mean & Min & Max & SD & Mean & Min & Max & SD & \\
\hline d FM/PICA (R) & 11.59 & 5.7 & 18.5 & 3.55 & 11.61 & 6.2 & 19 & 3.03 & -0.02 \\
\hline$\phi$ FMNA (R) & 2.65 & 1.2 & 3.4 & 0.46 & 2.49 & 1.2 & 3.2 & 0.47 & 1.68 \\
\hline d PICANBJ (R) & 12.66 & 6.4 & 19.4 & 3.38 & 12.28 & 7.7 & 18.3 & 2.22 & 0.69 \\
\hline$\phi$ PICANA (R) & 2.78 & 1.4 & 3.4 & 0.44 & 2.63 & 1.8 & 3.2 & 0.37 & 1.87 \\
\hline d V4 (R) & 24.25 & 12.1 & 36.8 & 6.76 & 23.89 & 14.7 & 37.3 & 4.89 & 0.32 \\
\hline$\phi$ VBJ & 3.24 & 2.1 & 4.2 & 0.58 & 3.24 & 2.1 & 4.3 & 0.61 & 0.02 \\
\hline$\alpha$ VBJ & 85.45 & 57 & 98 & 10.76 & 86.26 & 57 & 98 & 9.45 & -0.4 \\
\hline d FM/PICA (L) & 12.76 & 5.7 & 19.7 & 4.35 & 12.07 & 5.7 & 22.5 & 4.24 & 0.78 \\
\hline$\phi$ FM/NA (L) & 2.87 & 1.7 & 4.1 & 0.57 & 3.06 & 2 & 4.1 & 0.52 & -1.78 \\
\hline d PICANBJ (L) & 18.75 & 8.3 & 27.1 & 4.88 & 19.10 & 8.3 & 27.1 & 4.89 & -0.36 \\
\hline$\phi$ PICANA (L) & 3.16 & 1.9 & 4.2 & 0.63 & 3.25 & 2.1 & 4.3 & 0.59 & -0.75 \\
\hline dV4 (L) & 31.51 & 15.9 & 42.3 & 6.51 & 31.18 & 16.1 & 39.8 & 5.54 & 0.27 \\
\hline Age & 55.29 & 22 & 87 & 17.39 & 64.95 & 43 & 87 & 10.49 & -3.53 \\
\hline
\end{tabular}

$\mathrm{R}$ — right side of the vessel; $\mathrm{L}$ — left side of the vessel; $\mathrm{d}$ — length; $\phi$ — diameter; SD — standard deviation; rest abbreviation — see text

mean was $85.45 \pm 10.76^{\circ}$ in the group of women and $86.26 \pm 9.45^{\circ}$ in the group of men. There was not any statistical difference between the groups.

Hypoplasia was observed in the following amounts: at the level of foramen magnum on the right side in women (24.24\%) and in men (18.42\%); at the level of exit from the PICA on the right side in women (19.7\%) and in men (15.79\%); on the left side at the level of FM in women (19.7\%) and in men $(10.5 \%)$; at the level of the PICA in women (18.2\%) and in men $(13.16 \%)$, at the point of the VBJ in women (12.12\%) and in men (15.79\%).

In our series, ectasia was located at the level of entry of the VA to the intracranial space in women (19.7\%) and in men (13.16\%); at the level of exit from the PICA in women $(18.1 \%)$ and in men $(23.68 \%)$ on the left side. On the opposite, $\mathrm{V}_{4}$ ectasia was observed at the level of FM in women (13.64\%) and in men (13.16\%). At the level of PICA, there were $16.67 \%$ of ectasias in women and $13.16 \%$ in men. Ectasia was observed in the VBJ in women $(21.21 \%)$ and in men $(26.32 \%)$.

The following anatomical variations were also observed: 2 fenestrations, any case with duplication of $\mathrm{V}_{4}\left(1\right.$ in the segment of $\left.\mathrm{V}_{2}\right)$, any case of absent $\mathrm{V}_{4}$ in studying circulation, 7 PICA duplications, and 17 cases of absent PICA.

The percentage was considerably lower in our study and was calculated at the level: PICA duplication 3\% and $4 \%$ (left and right side), and PICA absent $9 \%$ and $8 \%$ (left and right side, respectively).

\section{DISCUSSION}

The vertebral arteries are an important source of blood supply to the neurological structures of the posterior cranial fossa. The typical course is divided into four segments and its location is well described in many anatomical articles derived from work performed on cadavers; however, their panorama was very difficult to investigate during surgery. The analysis of 3D angiography shows some advantages in this aspect and may be helpful in clinical diagnosis and determining how to treat pathology in this region. The more interesting part of the VA for neurosurgeons is $V_{4}$ where aneurysms are a significant surgical problem. Knowledge concerning anomalies and variations in the vertebrobasilar complex can be useful to radiologists in diagnosing associated aneurysms and in preventing complications during endovascular (coiling) procedures. Other diseases related to vascular pathologies, including trigeminal or glossopharyngeal neuralgia are described in medical journals in 4-5 per 100,000 of the world population. The cerebellopontine angle is the common location for tumours such as vestibular Schwannoma, meningioma, or epidermoid cysts, with an incidence of $8-10 \%$ of all intracranial neoplasms. Neurosurgeons must consider possible variations (fenestration, duplication, hypoplasia) in the VA complex before planning an operation in the posterior fossa [23]. Hypoperfusion of brain tissues and haemodynamic insufficiency due to hypoplasia or stenosis of the VA is the basis of a transient ischaemic attack or acute brainstem/cerebellar ischaemic stroke [2]. In light 
Table 2. Review of measurements of length, diameter and angle of connection to the vertebral artery

\begin{tabular}{|c|c|c|c|c|c|c|}
\hline $\begin{array}{l}\text { Author } \\
\text { [reference] }\end{array}$ & No. of exam. & Methodology & Total length & $\begin{array}{l}\text { Length form } \\
\text { PICA to VBJ }\end{array}$ & $\begin{array}{c}\text { Diameter } \\
\text { of PICA }\end{array}$ & Angle of VBJ \\
\hline Songur [23] & 110 & $\begin{array}{l}\text { Cadavers (dissec- } \\
\text { tion) brains }\end{array}$ & & & & $52.2 \pm 18.2^{\circ}$ \\
\hline Grand [12] & 28 & $\begin{array}{l}\text { Cadavers } \\
\text { (dissection) }\end{array}$ & & $\begin{array}{l}16.25(\mathrm{R}) \\
18.29(\mathrm{~L})\end{array}$ & $\begin{array}{l}1.23(0.8-2.5)(\mathrm{L}) \\
1.36(0.7-3.0)(\mathrm{R})\end{array}$ & \\
\hline Ballesteros [4] & 95 & $\begin{array}{l}\text { Cadavers } \\
\text { (dissection) }\end{array}$ & $\begin{array}{c}33.86 \pm 5.59(\mathrm{~L}) \\
32.47 \pm 4.8(\mathrm{R})\end{array}$ & & & \\
\hline
\end{tabular}

$\mathrm{L}$ — left side; PICA — posterior inferior cerebellar artery; $\mathrm{R}$ — right side; VBJ — vertebrobasilar junction

Table 3. Reports of measurements of the diameter and number of hypoplastic vertebral artery (VA)

\begin{tabular}{|c|c|c|c|c|c|c|c|}
\hline $\begin{array}{l}\text { Author } \\
\text { [reference] }\end{array}$ & $\begin{array}{l}\text { No. of } \\
\text { exam. }\end{array}$ & Methodology & $\begin{array}{c}\text { Diameter } \\
\text { of VA right side }\end{array}$ & $\begin{array}{c}\text { Diameter } \\
\text { of VA left side }\end{array}$ & $\begin{array}{l}\text { Hypoplasia } \\
\text { of right VA }\end{array}$ & $\begin{array}{c}\text { Hypoplasia } \\
\text { of left VA }\end{array}$ & $\begin{array}{c}\text { Bilateral } \\
\text { hypoplasia }\end{array}$ \\
\hline Akgun [1] & 188 & CT, MR imaging & $2.95 \pm 0.47$ & $3.23 \pm 0.57$ & & & \\
\hline Songur [23] & 110 & $\begin{array}{l}\text { Cadavers } \\
\text { (dissection) }\end{array}$ & $2.85 \pm 0.99$ & $3.02 \pm 0.81$ & $20.2 \%$ & $14.4 \%$ & $4.8 \%$ \\
\hline Grand [12] & 28 & $\begin{array}{l}\text { Cadavers } \\
\text { (dissection) }\end{array}$ & $2.45(1.0-4.0)$ & $2.72(1.5-4.5)$ & & & \\
\hline Duan [10] & 98 & Angio-CT & $3.0 \pm 0.72^{*}$ & $3.6 \pm 0.51^{*}$ & & & \\
\hline Ballesteros [4] & 95 & $\begin{array}{l}\text { Cadavers } \\
\text { (dissection) }\end{array}$ & $2.94 \pm 0.77$ & $3.12 \pm 0.85$ & $10.5 \%$ & $8.4 \%$ & \\
\hline Park [17] & 529 & MR angiography & & & $19.3 \%$ & $12.5 \%$ & $3.4 \%$ \\
\hline Deng [9] & 200 & MR angiography & $2.3 \pm 0.5$ & $2.6 \pm 0.5$ & & & \\
\hline
\end{tabular}

*The atlantoaxial and intracranial segments of $\mathrm{VA}\left(\mathrm{V}_{3}-\mathrm{V}_{4}\right)$; $\mathrm{CT}$ — computed tomography; $\mathrm{MR}$ - magnetic resonance

of such examples, a more extensive knowledge of the anatomical relation of the VA is extremely important to plan the appropriate surgical access.

The methodological quality of the analysed studies was extremely varied. The differences concern the following: materials (radiological exams or anatomical specimens), numbers of measured cases, types of imaging techniques (magnetic resonance angiography [MRA], CTA, duplex ultrasound, DSA), the segments of VA (more $V_{2}$, less $V_{4}$ ), heterogeneous cohorts (ill and healthy patients), dissimilar points of measurement on the $V_{4}$, no consensus of the definition of the "hypoplastic" vessel, and fenestration vs. "partial duplication".

The limitation of some previous articles includes the fact that all measurements were obtained using only one type of radiological examination (MRA or CTA or ultrasound) without control of another technique. In anatomical studies, there was a limited number of specimens and different methods of formalin fixation and artificial substance injection. In conclusion, this study provides comprehensive morphometric information concerning the construction of the vertebral circulation with a high component of clinical usefulness for multidisciplinary teams carrying out diagnostics and treatment of pathology located in the vicinity of VAs.

The results of the other anatomical studies are presented in Tables 2 and 3.

\section{The length and diameter of the $\mathbf{V}_{4}$}

Ballesteros et al. [4] reported that the intracranial part of VA has a mean total length of $33.86 \pm 5.59$ on the left and $32.47 \pm 4.8$ on the right side. The most common distance between the origin of the PICA to the VBJ is 16-18 mm [12]. Our calculations (the total and partial length with of $\mathrm{V}_{4}$ ) are presented in Table 1 . There were no statistically significant differences in the gender; however, the left artery was longer in both sexes.

A review of the literature showed the diameter of the $V_{4}$ from $1.5 \mathrm{~mm}$ to $4.5 \mathrm{~mm}$ using different measurement techniques (see Table 3 ). Unfortunately, there was no information about the location where the diameter was calculat- 
ed. These results are similar to ours (see Table 1) and are also consistent with the conclusions of the other studies that the left $\mathrm{V}_{4}$ is dominant more often than the right artery $[1,23]$. According to the published anatomical data, the left VA has a wider lumen and longer segment than the contralateral one in $50-70 \%$ of individuals $[15,19,21]$. Statistical differences in VA diameter between genders were not observed. However, Deng et al. [9] reported the opposite relation for the Chinese population in which males exhibited a significantly wider arterial lumen compared to that of females.

\section{Hypoplasia of the vertebral artery}

Infarcts of the vertebrobasilar circulation are responsible for $12-27 \%$ of all strokes in hospitalised patients, and $20-25 \%$ of these are believed to be due to stenosis of vertebral arteries $[15,19]$. Despite its apparent aetiological importance, optimal management (endovascular or surgical) of VA stenosis and other vasculopathies remains uncertain $[22,28]$.

Hypoplasia of $\mathrm{V}_{4}$ remains an uncommon embryogenic variation of the posterior circulation, which could have important haemodynamic implications (hypoperfusion and insufficient circulation) in acute brainstem/cerebellar ischaemic stroke $[17,19]$, or in the lateralisation of vestibular neuropathy [8]. In the pathoanatomical studies, hypoplastic VA is defined as a lumen diameter of $<3,<2.5,<2.2$ or $<2 \mathrm{~mm}$, and there is no consensus on this value $[7,19]$. The discrepancy results from the fact that studies were not always conducted on healthy subjects (many articles concern cases of ischaemic stroke), different methods of studies (autopsy, MRA, DSA, ultrasonography), and variable locations of diameter measurements. In some articles, colour-coded duple ultrasonography to visualise and calculate $V A$ parameters was limited to the cervical region $\left(V_{2}\right)$, thus, excluding the intracranial region $\left(V_{4}\right)$ [19]. There were some uncertainties of whether the measurements included external or internal diameters. Additionally, the parameters theoretically can change and depend on several factors including race, sex, age and the method of preservation of the brain tissue. For example, the diameter size may be dependent on the pressure which is exerted by a plastic mass such as latex while the blood vessel is being injected during the technique of filling the arteries [30]. Additional ambiguity in some clinical papers concerns the use of the terms "hypoplastic", "very small diameters", "abnormal" VA in the description of the operation or endovascular pro- cedure. However, such terms are not a strict definition of a vascular anomaly and may usually be a subjective feeling of the surgeons that are not based on radiological calculations. For example, many clinical papers show a different definition of hypoplasia.

Zmuda and Gielicki [30] proposed a definition of vessel hypoplasia based on the notion that the vascular asymmetry coefficient is the difference between the mean diameter vascular segments expressed as a percentage of the wider vessel with respect to the major diameter. Based on our previous analyses (where we proposed a mathematical definition of the posterior communicating arterial hypoplasia), we assumed that the parameter is the mean of the diameter reduced by 1 SD [11]. We observed a correlation between our definition of the hypoplastic posterior communicating artery and the lumen of the artery in patients who had suffered from cerebral ischaemic stroke due to ipsilateral internal carotid occlusion [11]. Therefore, the same definition of $\mathrm{V}_{4}$ hypoplasia was proposed. In our estimation, the hypoplasia was defined as ranging $10.5-24.24 \%$, depending on gender and the segment of $\mathrm{V}_{4}$. There was no statistically significant correlation between the sides of the vessel, gender, and the location of measurement, even though the variation was observed more frequently in the left artery. This is consistent with the suggestions of other authors of a dominant left VA with a wider lumen and longer segment than the contralateral one in $50-70 \%$ if individuals $[15,19,21]$.

\section{Vertebro-basilar junction - location and angle}

The level and geometry of the VBJ is particularly important with regard to selection of the surgical approach for aneurysm clipping in this region. Songur et al. [23] defined the location of the VBJ in relation to the bulbopontine sulcus and reported that the VBJ can be at the level of the sulcus in $20 \%$, below in $67 \%$, and above in $12 \%$ of cases [23].

Aneurysms located in the VBJ are fortunately relatively rare, and account for less than $4 \%$ of all vascular pathology located in posteriori circulation (there is a $0.5 \%$ incidence of intracranial aneurysms). The high risk of neurovascular complications during surgery makes endovascular treatment of such pathologies a widely accepted procedure.

Compared to other studies, the mean angle in the connection of the VA at the point of the basilar artery was much higher in our study (Table 1). Information concerning the relation between the angle and the risk of aneurysm is lacking. 


\section{Dolichoectasia}

The term dolichoectasia (derives from the Greek "dolicho" and "ectasia") means a local increase in the length and diameter. There may be an effect of congenital vasculopathy on the internal elastic layer with tunica media thinning being secondary to smooth muscle atrophy in the arterial wall and autoimmune process in InG4 related disease [24]. This vascular anomaly can be the cause of many neurological symptoms of the brainstem, cerebellar or cranial nerve dysfunction (especially hemifacial spasm and trigeminal neuralgia), pyramid pathology and hydrocephalus (although controversial) as well as haemorrhage and ischaemic stroke. Currently, there are no widely accepted criteria for the diagnosis of vertebraldolichoectasia, and it is often described in radiological examinations as a subjective judgement of the radiologist that the vessel is wider and longer than normal. There are different propositions of the minimum width of the lumen of the VA above which one can classify ectasia. Ubogu and Zaidat [25] suggested a VA diameter of less than $4.5 \mathrm{~mm}$, while Vasović et al. [27] suggested greater than $4.3 \mathrm{~mm}$, and others more than the mean diameter \pm SD [13]. These differences can arise from different medical methods used for analysis (cadavers or 3D MRA Medical Systems) and different points of measurement, such as the internal lumen used in radiological exams or the external lumen used in cadaver specimens. In the presented study, the mean diameter was less than $4 \mathrm{~mm}$ (shown in detail in Table 1). Our calculations were based on a more sensitive visualisation method (3D CTA or DSA in 3D space), and they were applied to the internal lumen in various places (contrast solution inside the artery). We took the cut point as the mean diameter \pm SD.

In our series, were identified from $13.16 \%$ to $26.32 \%$ ectasis depending on the location and side of the artery used for measurement. This ectasis percentage is much more than that reported previously (5.56\%) from measurements on human cadavers [27], or 1.6-4.4\% (both basilar artery and/or VA dolichoectasia) measured using MRA exams $[13,25]$. This anomaly was observed more often at the location of the division of the vessel, which is in a natural region of origin of saccular aneurysms. Therefore, these results may account for why dilatation is regarded as a risk factor for aneurysm. Based on the results of previous studies, we accepted that the VA was considered elongated if the length was $>23.3 \mathrm{~mm}$ [25]. According to our measurements, $50 \%$ of the left VA vessels and $84.85 \%$ on the right side in women were longer than this limit. A similar situation was observed in men with $69.44 \%$ longer left vessels and $94.44 \%$ lon- ger right segments. On the other hand, the anatomical studies showed similar total intracranial lengths of $\mathrm{V}_{4}$ at 33.86-32.47 mm (Table 2). Therefore, we only use the term ectasia and not dolichectasia. The literature does not show a consensus about the dependence of dolichoectasia and gender. Some authors described the presence of dolichoectasia mainly in males and others in female patients. We did not find a statistical relationship between sex and vascular ectasia, or between the side (right/left) and vascular ectasia. In conclusion, vertebrobasilar dolichoectasia is a potential severe condition that may cause severe neurological disability. There is a problem with its recognition by radiologists and it pathological classification and not to the normal variation in the tortuosity or calibre of the artery [25].

\section{The anatomical variations (fenestration, duplication, absence)}

Akgun et al. [1] showed on the basis of radiological exams (64-detector row CT and 3T MRA) that only $34.8 \%$ cases in the studied group had a well-known "normal" vertebrobasilar system anatomy. Other authors emphasise that asymmetric VAs occur in over 2/3 of individuals [19]. The anatomical variations of the circulation include: hypoplasia, aplasia or absent artery, fenestrations, duplications, persistent foetal arteries, dolichoectasia, abnormal variations of course, collateral branches, and crossing by VAs. These variations necessitate the improvement of our knowledge about vascular morphometry in the clinical context.

Similar to the various definitions of hypoplasia, there are alternative names for fenestration and duplication in the medical literature. Fenestration is otherwise described as "partial" or "segmental duplication", and duplication is also referred to as "extreme fenestration" [20]. Fenestration of the artery is a rare congenital variation normally related to the abnormal development of primitive embryonic vessels and may form as the result of persistence of the arterial collateral system; however, the aetiology of this variation remains unclear. Fenestration can be due to the failure of complete fusion of the two neural arteries of the embryo between the $29^{\text {th }}$ and $30^{\text {th }}$ day of foetal development [20]. The term "fenestration" is reserved for this variation with a common origin, and the vessel subsequently splits into two parallel channels, which re-join distally [5]. The arterial walls have their own endothelial and muscular layers while the adventitia may or may not be shared [3].

Duplication is a condition in which an artery has two origins and one fusion (exit point) or in other words two 
distinct arteries have separate origins without distal arterial convergence [3]. According to other publications, the duplication can form due to the failure of regression of primitive vessels during embryological development between the $32^{\text {nd }}$ and $40^{\text {th }}$ day, and fenestration is due to failure of the complete fusion of the two neural arteries of the embryo $[6,20]$.

Some authors agree with a hypothesis that duplication and fenestration predispose the patient to coexisting vascular anomalies (aneurysms, vascular malformations, vertebral dissection etc.) $[21,26,29]$. Such vascular anomalies may result from changes in the construction of the vascular wall and altered haemodynamics (the turbulent blood flow) [3]. The tunica media at the proximal and distal ends of the duplicated segment have an irregular pattern or a complete absence of elastic fibres, which may account for the symptoms of posterior circulation insufficiency in patients. The recognition of this anomaly is especially important for planning the catheter route during endovascular examination or treatment. There are some results that do not support the hypothesis presented above $[5,7,14]$. The author documented the relation between fenestrations in all intracranial arteries (not only in VAs) vs. aneurysms, suggesting that fenestration is recognised as a risk for aneurismal formation but the significant association is not yet established [14]. It has also been shown that the rate of fenestration is not significantly different in cases with and without aneurysms, and conversely the rate of aneurysms in not significantly different in patients with or without fenestration [7].

The incidence of VA fenestration is described in the literature in the range from $0.23 \%$ to $2.2 \%$ on the basis of different radiological examination (DSA, MRA, CTA) $[5,7,16]$ and was predominant in the female group in all measured intracranial arteries (not only VA) [5]. In our series, there were two fenestrations (one on both sides) in the segment of $\mathrm{V}_{4}$.

The frequency of duplication was identified by Bergamn et al. [6] in $0.72 \%$ of autopsy studies; however, more than $2 / 3$ were located in the extracranial segment of the VA and all were situated on the left side. In our material, we did not observe any cases of duplication of the $\mathrm{V}_{4}$ segment; however, we observed one case of duplication in the right artery, which formed a connection in the section $V_{2}$.

The absence of some part of the VA is a very rare embryological anomaly, and its estimated incidence is from $1.05 \%$ to $4.4 \%[1,4,14,18,29]$. In the cited literature, Akgun et al. [1] showed 1 case of the left VA and 3 cases of right VAs which were ending as PICA. Kovać et al. [14] observed this anomaly in 9 patients (they examined 455 patients using (TA), and transversal anastomosis between VAs was observed in only 1 case. Zhou et al. [29] described 3 patients with hypolpastic VAs terminating in PICA or a common PICA/AICA trunk, and Pekcevik and Pekcevik [18] described 15 (4.4\%) such vascular anomalies in 341 patients examined by 64-slice CT. Ballesteros et al. [4] ascertained 1 case of VA agenesis of large calibre on the opposite side (the same as the proximal segment of the basilar artery) from analysing 95 fresh cadaver specimen autopsies. In our study, we did not observe any cases of absence of VAs in the circulation.

Duan et al. [10], using 3D CT examination on 98 patients, reported a duplication of the PICA in $15.31 \%$ of cases on the left side and $7.14 \%$ on the right. An absence of the PICA was noted in $2.3-6 \%$ of cases bilaterally and $19.3-25.5 \%$ unilaterally $[1,10]$. The percentage of anatomical variations of the PICA was calculated in our study as follows: 3 (4\%) duplications and $9(8 \%)$ cases of absent PICA.

In summary, there was no correlation between gender and anatomical variations regarding fenestration, duplication or absence in the presented study. There are two general clinical facts concerning our results. First, possible variations in the VAs should be considered by neurosurgeons before planning an operation in the posterior cranial fossa. Second, radiologists must also consider arterial variations regarding DSA, CTA, and MRA during exam descriptions and while planning endovascular procedures.

\section{CONCLUSIONS}

The anatomical information obtained from 3D CT scans and DSA reconstructions is very helpful in the radiological diagnosis of $\mathrm{V}_{4}$ diseases and in anatomical analyses with the elements of practicality for clinical applications. The presented documentation of anatomical variation and abnormalities of the vertebral circulation can improve the accuracy and "safety" of surgical procedures in this region. Such information facilitates decisions about the range of surgical approach and helps to avoid associated complications.

Radiological examinations using 3D CT and DSA provide extensive information about anatomical structures in contrast to cadaver-based studies, and can complement the morphometry in anatomical preparations.

The left $V_{4}$ is predominant over the right artery, which is manifested by the length, the width, cases of ectasis and fewer cases of hypoplasia. The incidences of $V_{4}$ ectasis were identified more often than reported 
in the accessible literature and they were observed in the natural place of formation of saccular aneurysms.

\section{REFERENCES}

1. Akgun V, Battal B, Bozkurt $Y$, et al. Normal anatomical features and variations of the vertebrobasilar circulation and its branches: an analysis with 64-detector row CT and 3T MR angiographies. Sci World J. 2013; 2013: 620162, doi: 10.1155/2013/620162, indexed in Pubmed: 24023533.

2. Amin-Hanjani S, Rose-Finnell L, Richardson D, et al. VERiTAS Study Group. Vertebrobasilar Flow Evaluation and Risk of Transient Ischaemic Attack and Stroke study (VERiTAS): rationale and design. Int J Stroke. 2010; 5(6): 499-505, doi: 10.1111/j.1747-4949.2010.00528.x, indexed in Pubmed: 21050408.

3. Arráez-Aybar LA, Villar-Martin A, Poyatos-Ruiperez C, et al. Prevalence of fenestrated basilar artery with magnetic resonance angiography: a transversal study. Surg Radiol Anat. 2013; 35(6): 487-493, doi: 10.1007/s00276-012-1053-5, indexed in Pubmed: 23250566.

4. Ballesteros L, Forero P, Quintero I. Morphological expression of the anterior spinal artery and the intracranial segment of the vertebral artery: a direct anatomic study. Rom J Morphol Embryol. 2013; 54(3): 513-518, indexed in Pubmed: 24068398.

5. Bayrak AH, Senturk S, Akay HO, et al. The frequency of intracranial arterial fenestrations: a study with 64-detector CT-angiography. Eur J Radiol. 2011; 77(3): 392-396, doi: 10.1016/j. ejrad.2009.09.015, indexed in Pubmed: 19811882.

6. Bergman RA, Thompson SA, Afifi AK, Saadeh FA. Compendium of anatomic variation: catalog, atlas and world literature. Urban \& Schwarzenberg, Baltiomore-Munch 1988.

7. Bharatha A, Aviv RI, White J, et al. Intracranial arterial fenestrations: frequency on CT angiography and association with other vascular lesions. Surg Radiol Anat. 2008; 30(5): 397-401, doi: 10.1007/ s00276-008-0340-7, indexed in Pubmed: 18350245.

8. Chuang YM, Chern CM, Liao WH, et al. Contribution of intracranial vertebral artery asymmetry to vestibular neuropathy. J Neurol Neurosurg Psychiatry. 2011; 82(7): 823-825, doi: 10.1136/ jnnp.2009.203323, indexed in Pubmed: 20587480.

9. Deng D, Cheng FuBo, Zhang Y, et al. Morphological analysis of the vertebral and basilar arteries in the Chinese population provides greater diagnostic accuracy of vertebrobasilar dolichoectasia and reveals gender differences. Surg Radiol Anat. 2012; 34(7): 645-650, doi: 10.1007/s00276-012-0960-9, indexed in Pubmed: 22427028.

10. Duan S, He H, Lv S, et al. Three-dimensional CT study on the anatomy of vertebral artery at atlantoaxial and intracranial segment. Surg Radiol Anat. 2010; 32(1): 39-44, doi: 10.1007/ s00276-009-0552-5, indexed in Pubmed: 19707709.

11. Dzierżanowski J, Szarmach A, Słoniewski P, et al. The posterior communicating artery: morphometric study in 3D angio-computed tomography reconstruction. The proof of the mathematical definition of the hypoplasia. Folia Morphol. 2014; 73(3): 286-291, doi: 10.5603/FM.2014.0045, indexed in Pubmed: 25242155.

12. Grand W, Budny JL, Gibbons KJ, et al. Microvascular surgical anatomy of the vertebrobasilar junction. Neurosurgery. 1997; 40(6): 1219-23; discussion 1223, indexed in Pubmed: 9179895.

13. Ikeda K, Nakamura $Y$, Hirayama T, et al. Cardiovascular risk and neuroradiological profiles in asymptomatic vertebrobasilar dolichoectasia. Cerebrovasc Dis. 2013; 30(1): 23-28, doi: 10.1159/000313440, indexed in Pubmed: 20424441.

14. Kovač JD, Stanković A, Stanković D, et al. Intracranial arterial variations: a comprehensive evaluation using $C T$ angiography. Med Sci Monit. 2014; 20: 420-427, doi: 10.12659/MSM.890265, indexed in Pubmed: 24625840.
15. Nouh A, Remke J, Ruland S. Ischemic posterior circulation stroke: a review of anatomy, clinical presentations, diagnosis, and current management. Front Neurol. 2014; 5: 30-41, doi: 10.3389/ fneur.2014.00030, indexed in Pubmed: 24778625.

16. Ozpinar A, Magill ST, Davies JM, et al. Vertebral Artery Fenestration. Cureus. 2015; 7(1): 245-249, doi: 10.7759/cureus.245, indexed in Pubmed: 26180669.

17. Park JH, Kim JM, Roh JK. Hypoplastic vertebral artery: frequency and associations with ischaemic stroke territory. J Neurol Neurosurg Psychiatry. 2007; 78(9): 954-958, doi: 10.1136/ jnnp.2006.105767, indexed in Pubmed: 17098838.

18. Pekcevik Y, Pekcevik R. Variations of the cerebellar arteries at CT angiography. Surg Radiol Anat. 2014; 36(5): 455-461, doi: 10.1007/ s00276-013-1208-z, indexed in Pubmed: 24061702.

19. Perren F, Poglia D, Landis T, et al. Vertebral artery hypoplasia: a predisposing factor for posterior circulation stroke? Neurology. 2007; 68(1): 65-67, doi: 10.1212/01.wnl.0000250258.76706.98, indexed in Pubmed: 17200496.

20. Ples H, Loukas M, lacob N, et al. Duplication of the distal end of the left vertebral artery with fenestration of the right posterior cerebral artery. Rom J Morphol Embryol. 2015; 56: 575-577.

21. Polguj $M$, Podgórski $M$, Jędrzejewski $K$, et al. Fenestration and duplication of the vertebral artery: the anatomical and clinical points of view. Clin Anat. 2013; 26(8): 933-943, doi: 10.1002/ ca.22231, indexed in Pubmed: 23553773.

22. Shi X, Qian H, Singh KC, et al. Surgical management of vertebral and basilar artery aneurysms: a single center experience in 41 patients. Acta Neurochir (Wien). 2013; 155(6): 1087-1093, doi: 10.1007/s00701-013-1656-6, indexed in Pubmed: 23471600.

23. Songur A, Gonul $Y$, Ozen $O A$, et al. Variations in the intracranial vertebrobasilar system. Surg Radiol Anat. 2008; 30(3): 257-264, doi: 10.1007/s00276-008-0309-6, indexed in Pubmed: 18253692.

24. Toyoshima Y, Emura I, Umeda Y, et al. Vertebral basilar system dolichoectasia with marked infiltration of IgG4-containing plasma cells: a manifestation of IgG4-related disease? Neuropathology. 2012; 32(1): 100-104, doi: 10.1111/j.1440-1789.2011.01227.x, indexed in Pubmed: 21615518.

25. Ubogu EE, Zaidat OO. Vertebrobasilar dolichoectasia diagnosed by magnetic resonance angiography and risk of stroke and death: a cohort study. J Neurol Neurosurg Psychiatry. 2004; 75(1): 22-26, indexed in Pubmed: 14707300.

26. Uchino A, Saito N, Okada Y, et al. Fenestrations of the intracranial vertebrobasilar system diagnosed by MR angiography. Neuroradiology. 2012; 54(5): 445-450, doi: 10.1007/s00234-011-0903-x, indexed in Pubmed: 21732084.

27. Vasović L, Trandafilović M, Jovanović I, et al. Vertebral and/or basilar dolichoectasia in human adult cadavers. Acta Neurochir (Wien). 2012; 154(8): 1477-1488, doi: 10.1007/s00701-0121400-7, indexed in Pubmed: 22664729.

28. Zang $Y$, Wang $C$, Zhang $Y$, et al. Long-term follow-up study of 35 cases after endovascular treatment for vertebrobasilar dissecting aneurysms. Clin Neurol Neurosurg. 2015; 137: 121-131, doi: 10.1016/j.clineuro.2015.07.004, indexed in Pubmed: 26196477.

29. Zhou M, Zheng H, Gong S, et al. Vertebral artery hypoplasia and vertebral artery dissection: a hospital-based cohort study. Neurology. 2015; 84(8): 818-824, doi: 10.1212/WNL.0000000000001284, indexed in Pubmed: 25632095.

30. Zmuda A, Gielecki JS. A novel formula for the classification of blood vessels according to symmetry, asymmetry and hypoplasia. Folia Morphol. 2007; 66(4): 339-345, indexed in Pubmed: 18058758. 